\title{
Imaging Diagnosis of Hepatocellular Carcinoma: Recent Advances of Contrast-Enhanced Ultrasonography with SonoVue ${ }^{\circledR}$
}

\author{
Veronica Salvatore Alice Gianstefani Giulia Negrini \\ Giulia Allegretti Marzia Galassi Fabio Piscaglia \\ Division of Internal Medicine, Department of Medical and Surgical Science, University of Bologna, \\ S.Orsola-Malpighi Hospital, Bologna, Italy
}

\author{
Key Words \\ Cholangiocarcinoma $\cdot$ Cirrhosis · Diagnosis $\cdot$ Hepatocellular carcinoma $\cdot$ Treatment
}

\begin{abstract}
Due to the ability to detect the typical contrast-imaging pattern for hepatocellular carcinoma (HCC), that is hyperenhancement in the arterial phase and hypoenhancement in the late phase on a cirrhotic background, contrast-enhanced ultrasonography (CEUS) was included in the American diagnostic algorithm for HCC in 2005. However, its role has been questioned because of the possibility of misdiagnosis of cholangiocarcinoma. The present review aims to describe the advantages and disadvantages of CEUS applications using Sonovue ${ }^{\circledR}$ for HCC. In particular there is focus on the accuracy of CEUS in detecting the typical HCC pattern, the CEUS patterns of intrahepatic cholangiocarcinoma (ICC), the risk of misdiagnosis with HCC, the diagnostic use of CEUS in cases of locoregional and systemic treatments, and the evaluation of response to antiangiogenic treatment using dedicated software.
\end{abstract}

Copyright $\odot 2015$ S. Karger AG, Basel

\section{Introduction}

Ultrasonography (US) has substantially impacted the way to manage liver cancer since its introduction in the 1980's. In particular the arrival of real time grey scale conventional 
US at that time not only permitted the detection of small lesions, but also allowed the development of ultrasound-guided techniques such as biopsy and ablation. The introduction of Doppler ultrasound followed shortly later in the late 1980's.

However, not much had changed in the field of US in the last 15-20 years, when an enormous step forward was made with the arrival of the first second-generation US contrast agent, sulphur exafluoride (SonoVue ${ }^{\circledR}$ ) and ultrasound machines with contrast specific software in 2002. Contrast-enhanced ultrasonography (CEUS), as a technique, was defined by the first guidelines of the European Federation of Societies for Ultrasound in Medicine and Biology (EFSUMB) [1]. It dramatically changed the ability to diagnose and manage of hepatocellular carcinomas (HCC) due to the ability to detect vascular lesion perfusion in real time, in addition to the advantages of the absence of ionizing radiation, a very high degree of safety, a relatively low cost, and easy repeatability [2]. With the ability to identify hyperenhancement in the arterial phase and hypoenhancement in the late phase (on a cirrhotic background) in HCC [3], CEUS was included in the American diagnostic algorithm for HCC in 2005 [4]. However, its role has been questioned because of the possibility of misdiagnosis of cholangiocarcinoma. The present review aims to describe advantages and disadvanatages of CEUS applications using SonoVue ${ }^{\circledR}$ (Bracco Imaging, Italy) for HCC, that have emerged in the last 5 years from 2009-2014.

A short description of the status of CEUS with SonoVue ${ }^{\circledR}$ in focal lesions in cirrhosis, as of 2009, will be reported. An analysis of the literature of the last 5 years from 2009-2014, will be presented with specific reference to the following points: i) accuracy of CEUS (predictive values for HCC of different CEUS patterns and their sensitivities), ii) CEUS patterns of intrahepatic cholangiocarcinoma (ICC) and the risk of misdiagnosis with HCC, with a critical review of the issue, iii) diagnostic use of CEUS in cases of locoregional treatments, and iv) the use of CEUS to diagnose the response to antiangiogenic treatment with sorafenib in HCC.

\section{Overview of Ceus Usage Status as of 2009}

The multistep hepatocarcinogenesis in cirrhosis, which starts from macroregenerative nodules and proceeds through low- and high-grade dysplastic nodules to well-, moderately- and poorly-differentiated HCC is characterized by a progressive increase in the newly formed tumor arterial supply and a decrease in the normal supply from "paired" arteries and the portal sysytem [5-9]. Due to these vascular modifications, a noninvasive diagnosis of HCC, by multiphase contrast imaging techniques such as computed tomography (CT) and magnetic resonance imaging (MRI), has become possible in liver cirrhosis. These diagnostic techniques were incorporated in the first release of the European guidelines in 2001 [10], and unlike malignancies in other organs, there was no requirement for pathological confirmation. Following the introduction of CEUS, it was demonstrated that this technique was also able to detect the hallmark pattern of HCC - hyperenhancement in the arterial phase followed by wash-out (fig. 1). As a result of the high sensitivity of this technique [3], CEUS was introduced in the American diagnostic algorithm for HCC in 2005 [4], and it was endorsed in the guidelines of the EFSUMB as a diagnostic method for HCC [11]. The very high positive predictive value (PPV) of the typical CEUS HCC pattern, which allowed its introduction in the guidelines based on expert opinion, was also later confirmed by prospective studies, having histological confirmation as a reference standard $[12,13]$. By 2009, the Japanese guidelines [14] had not included CEUS since no US contrast agent had been introduced in Japan at that time. Conversely, the Asian-Pacific guidelines, drafted in 2008 and published in 2010, included CEUS, but only with Sonazoid ${ }^{\circledR}$ or Levovist ${ }^{\circledR}$, and not SonoVue ${ }^{\circledR}$, as the latter had 


\section{Liver}

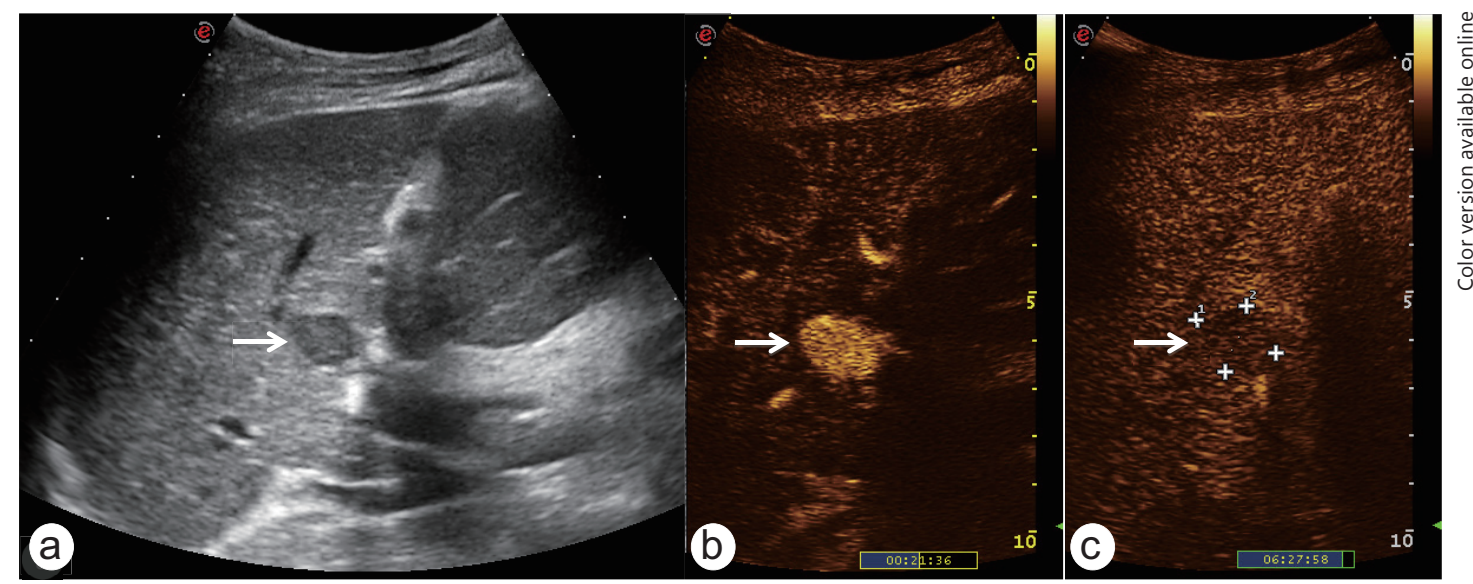

Fig. 1. Typical HCC pattern. CEUS of a hypoechoic lesion (arrow) in cirrhosis (a) demonstrates a marked hyperenhancement in the arterial phase (b) followed by wash-out in the late venous phase (c).

not been introduced in those countries [15]. The latest release of the American guidelines in 2009, corresponding to those published in 2005, accepted CT, MRI or CEUS as diagnostic for HCC with the following specifications: a single noninvasive imaging technique is sufficient for lesions $>2 \mathrm{~cm}$ in diameter, whereas two imaging techniques are required for lesions in the 1-2 cm diameter range $[4,16]$. Just how sensitive CEUS was for the detection of the complete hallmark pattern emerged as a critical issue, with initial data showing sensitivities in HCC ranging from 22 to $50 \%$ for small nodules $(<3 \mathrm{~cm}$ in diameter) $[12,13,17]$. A question about whether these limited sensitivities, usually 10-20\% lower than with CT and MRI $[12,13,17]$, was related to the degree of tumor differentiation in small nodules (frequently well differentiated in contrast to larger nodules which are more often moderately or poorly differentiated) was put forward based on some initial reports [18-20], but no definitive answer was found. Nonetheless, the regular use of CEUS in the diagnostic flowchart in combination with either CT or MRI appears economically advantageous for nodules sized 1-2 $\mathrm{cm}$ in diameter in comparison to the combination of CT with MRI after the first detection of such nodules in cirrhotic patients [21]. The AASLD 2005 guidelines state that investigations of HCCs should include at least one panoramic radiology technique (either CT or MRI) to stage the malignant disease, and the need for two positive imaging techniques for nodules $<2 \mathrm{~cm}$ in diameter [4]. On this basis, CEUS with SonoVue ${ }^{\circledR}$ has become largely utilized in cirrhotic patient in many countries in which this contrast agent had been introduced, and new studies have dealt with the sensitivity and specificity of CEUS for HCC and the risk of misdiagnosis of ICC. Together with its widespread uptake by physicians, many authors started to investigate the utility of CEUS in detecting residual activity after percutaneous treatments, mainly in order to refine incomplete treatments, an approach nowadays widely used and later discussed herein.

\section{Recent Reports about the Diagnostic Accuracy of Ceus in Focal Lesions in Cirrhosis}

The Sensitivity of Ceus for the Typical Hallmark Pattern Is Related to Size of the Lesion

The diagnostic value of CEUS in the evaluation of focal liver lesions (FLLs) has been evaluated by Ryu et al. [22] Their study was a retrospective analysis of 48 patients with and without liver disease. Using criteria described in the EFSUMB guidelines with respect to typical 
enhancement pattern of FLLs, CEUS was compared with contrast-enhanced CT (CECT) and contrast-enhanced MRI (CEMRI), respectively. The sensitivity, specificity and accuracy of 50 masses ( 43 HCCs, 3 hemangiomas, 2 adenomas, 1 eosinophilic abscess and 1 metastasis) was respectively $83 \%, 88 \%$ and $84 \%$ for CEUS, $95 \%, 88 \%$ and $94 \%$ for CECT and $95 \%, 83 \%$ and $93 \%$ for CEMRI. Interestingly, the majority of FLLs that were discordant among techniques, with failure of US, were undetectable at CEUS due to their location at the hepatic dome., A subanalysis excluding lesions with a poor acoustic window, demonstrated an accuracy of 93\%, like CECT and CEMRI [22], and in line with the rate of $90 \%$ reported by Strobel et al. in their study of 1,349 FLLs [23]. Whilst the limited number of patients affect the power of the study, this investigation emphasizes the limitations of CEUS with regard to FLL location, also already largely acknowledge by the guidelines [11].

An overall sensitivity of $88 \%$ for CEUS in diagnosing HCC, regardless of the presence of cirrhosis, emerged in a recent meta-analysis [24] of 2238 patient with the disease. However, the inclusion of patients with and without liver cirrhosis limits these results because the diagnostic approach in these two scenarios is totally different.

The HCC perfusion pattern has been widely investigated. When the dimensions are not taken into account for subgrouping, HCC showed hyperenhancement in the arterial phase in $93 \%$ and hypoenhancement in the late phase in $97 \%$ of cases [25]. Unfortunately, these findings, which were calculated on a series of large tumors with a mean diameter of $5.7 \mathrm{~cm}( \pm \mathrm{SD}$ $3.0 \mathrm{~cm}$ ranging from 1.4 to $14.9 \mathrm{~cm}$ ), are not well reproduced when nodules are stratified according to the lesion size. Indeed, the HCC pattern is not always typical in small lesions. The discrimination between neoplastic and non-neoplastic lesions on a cirrhotic background was evaluated by Xu et al. [26], who analysed 225 consecutive patients during a US imaging surveillance program. Based on the typical enhancement pattern, the accuracy of CEUS was $91 \%$, with a PPV of $100 \%$ and a negative predictive value (NPV) of $70.5 \%$, respectively. However, the accuracy increased with lesion diameter, reaching $97.3 \%$ in $2.1-3.0 \mathrm{~cm}$ diameter lesions and $100 \%$ in 3.1-5.0 cm diameter lesions. Surprisingly, their results were even better than those of previous studies that demonstrated that only half of HCCs smaller that $2 \mathrm{~cm}$ in diameter are hypervascular at angiography [27] or at CEUS [28]. The detection of hyperenhancement in the arterial phase without 'wash-out' (an event that took place only in $6 \%$ of their records, observed until 6 minutes and including 7 HCC, 3 high grade dysplastic nodules and one macro-regenerative nodule), is still debated in terms of the need to treat such lesions without obtaining a preliminary histological confirmation. Conversely, only $39 \%$ of nodules smaller than $2 \mathrm{~cm}$ diameter showed hyperenhancement in the arterial phase by CEUS compared to 34\% at CECT, according to Quaia et al. [29] Forner et al. found that $15 \%$ of study subject lesions showed hyperenhancement [30], despite that many of those not hyperenhancing in the arterial phase were nonetheless still HCCs. Interestingly, none of these hypovascular lesions were poorly differentiated. These data confirm a potential malignant nature of nodules even in absence of hyperenhancement in the arterial phase, not supporting a "wait-and-see" approach in this context.

The Degree of Venous Wash-Out Is Inversely Associated with the Grade of Tumor Differentiation

The accuracy of CEUS is greatly impacted by the tumor grade [31]. The typical pattern is present in only $17 \%$ of grade 1 HCCs and in $41 \%$ of grade $2-3$ HCCs. Accordingly, atypical patterns have been described especially in cases of well differentiated HCCs, that may be isoenhancing in the late phase $[18,32,33]$, or may present with wash-out, and in half of the cases occurring only later than three minutes [20]. The grading of HCCs according to their perfusion pattern can be inferred with a positive diagnostic likelihood ratio of 18.2 for predicting G2-G3 (moderately or poorly differentiated) differentiation grades, and a negative 
likelihood ratio of 0.06 [32], respectively. Indeed, higher grades of tumor differentiation (G2 or G3) are more often hypoenhanced in the portal/late phase whereas the majority of lesions that do not present with wash-out are more often grade G1 (well differentiated) HCCs [32].

To summarize, the diagnostic accuracy of CEUS is affected by the tumor dimensions and grade, two characteristics that are often interrelated; namely, smaller lesions are more often well differentiated [34]. Thus, the detection of an atypical CEUS pattern of FLLs on a background of cirrhosis dictates that nodule dimensions must be accounted for and one should pursue a histological diagnosis whenever feasible, because such early HCCs have the highest probability to receive curative treatments, differently from when they become larger.

\section{The Issue of Misdiagnosis with Cholangiocarcinoma}

In 2010, Vilana and colleagues from the Barcelona Clinic Liver Cancer [35] described the CEUS pattern of 21 ICCs that were present in cirrhotic patients, showing that 10 of them (48\%) had an appearance consistent with global arterial hyperenhancement and portal-venous wash-out, corresponding to the pattern which was until that point, typical only for HCCs. Conversely, MRI studies did not show such wash-out phenomenon, hence avoiding the risk of misdiagnosis. However, this prevented the possibility to establishing a diagnosis of malignancy by imaging alone. As a result, the EASL and AASLD guidelines from 2010 onwards, excluded CEUS from the diagnostic flow chart due to a possible misdiagnosis of ICC [35, 36]. In a study by Lu et al., [37] they demonstrated that $62.5 \%$ of ICCs $>2 \mathrm{~cm}$ in diameter occurring on a background of cirrhosis showed an enhancement pattern similar to HCCs. Moreover, they stratified the patients according to the presence of cirrhosis or healthy background liver and interestingly, the pattern described as typical for ICCs (rim-like/non-enhancement in the arterial phase followed by hypo/non-enhancement in late phase) [11] was more frequently identified in ICCs in normal livers. These data are in keeping with a recent study which also evaluated the relationship between CEUS patterns and the histological characteristics of ICCs, which reported similar results [38]. A retrospective study [39] analyzed 33 histologically proven ICCs in cirrhotic patients compared to 50 randomly selected histological proven HCCs. No difference between ICCs and HCCs was established in terms of time of contrast appearance in the tumor or in the proportion of homogeneous hyperenhancement and heterogeneous hyperenhancement in the arterial phase. The only difference was in terms of the peak intensity of tumor and tumor/tissue ratio, which was lower in ICCs. Concerning the portal phase, ICCs showed earlier wash-out, in the majority of cases within 60 seconds (88\%), whereas HCCs showed wash-out later than 60 seconds in $84 \%$ of cases, respectively. Moreover, a marked wash-out was noted in $79 \%$ of ICCs and only $12 \%$ of HCCs. Lastly, in the late phase, $91 \%$ of ICCs showed marked wash-out when compared to $44 \%$ of HCCs. Thus, the diagnostic accuracy of CEUS was $84 \%$ if early and marked wash-out in the late part of the portal phase was considered as typical [39]. These results are slightly different from Vilana et al. [35] and Galassi et al. [40] concerning the rate of ICCs that showed early wash-out, probably due to the larger rate of hepatitis B virus-related cirrhosis and due to a larger mean \pm SD mass diameter (49.9 $\pm 27.1 \mathrm{~mm}$ versus $30.4 \pm 11.6 \mathrm{~mm}$ ). Indeed, when only small ICCs (within the Milano Criteria) were included, a marked wash-out was evident in only $24 \%$ of ICCs [40]. However, Lu and coworkers reported the median time to onset of wash-out in arterially hyperenhanced ICCs of only 43 seconds and of less than 1 minute (50 seconds) in HCCs $<3 \mathrm{~cm}$ in diameter [38].

The updated EASL and AASLD recommendations $[16,41]$ decreased the lesion threshold for single imaging diagnosis from 2 to $1 \mathrm{~cm}$ in diameter, and CEUS was abandoned. Following these modifications, a sequential approach was suggested from a cost-effective point of view. This approach envisaged the use of CEMRI in cases when CECT was non-diagnostic for nodules $>1 \mathrm{~cm}$ in diameter, and fine needle biopsy in cases with a lack of a typical pattern after utilizing both of these imaging techniques [42]. However, this optimal scenario encountered 
real clinical practice in which MRI is not feasible in all patients mainly due to its limited availability, costs, presence of contraindications or factors limiting the technical quality, such as insufficient patient cooperation in breath holding. Therefore, the approach suggested by the Italian Society for the Study of the Liver and the British National Institute for Health and Clinical Excellence, recommends CEUS in selected cases which are not appropriate, not accessible or unavailable for the use of CEMRI $[43,44]$.

\section{Guidance and Evaluation of HCC Treatments}

CEUS has been also used to guide percutaneous treatments including radiofrequency (RF), percutaneous ethanol injection (PEI) and microwave ablation (MWA), mainly to overcome mistargeting, an event that may occur in $2 \%$ of cases [45]. Indeed, the differentiation between a neoplastic nodule and the cirrhotic background on B-mode US has been reported to be only about $75 \%$ in HCCs $<3 \mathrm{~cm}$ in diameter detected by CECT or CEMRI [46]. Visualization of nodules can be improved by contrast injection that can be performed immediately before or during the treatment. A limitation of SonoVue ${ }^{\circledR}$ with respect to Sonazoid ${ }^{\circledR}$ is represented by the relatively short time of microbubble persistence (usually about 4-6 minutes) that can make it difficult for the simultaneous insertion of the needle. However, even in cases of using CEUS, technical failure in recognizing lesions that are poorly identifiable on B-mode US ranges from $2 \%$ to $11 \%[47,48]$ depending on lesion size. The response to percutaneous treatment performed under CEUS guidance appears to be appealing according to the results presented by Rajesh et al. [49] who reported no residual recurrence during a mean follow up of 16 months (range 9-26 months).

Beside its role in guiding percutaneous treatments, CEUS can also be used to evaluate the response to treatments. Incomplete necrosis after locoregional treatment is associated with a poor long-term survival [50]. Residual viable tumor foci should be suspected in the instance of enhancement in a portion of a treated lesion. Even though the response to locoregional treatment is usually assessed by means of CT or MRI, the EFSUMB guidelines suggest to use CEUS due to its high accuracy (91-100\%), when CT or MRI are contraindicated or inconclusive [11, 51-54]. Additionally, the possibility to detect residual viable tumor portions shortly after an ablation procedure allows re-treatment during the same session. However, it is worth remembering that an immediate assessment of the deeper portion of a lesion is limited by gas formation or cavitation in cases of RF or by the presence of ethanol in cases of PEI. A time span of 24 hours between the treatment and CEUS would allow for optimal visualization, but it would prevent immediate re-treatments; for this reason, CEUS is routinely performed only after 10 minutes when the majority of artifacts have disappeared [2]. False positive findings for HCC persistence may occur in the first few weeks after RF ablation due to the presence of a hyperemic halo or to the occurrence of arteriovenous fistulas. However, in postablation reactions the enhancement observed during the arterial phase persists also during the portal and venous phases, without the occurrence of wash-out. Provided that venous wash-out is present before ablation, this feature may be of value [51]. A particular enhancement named "linear enhancement" has been noted [55], and is described as a linearshaped enhancement that runs from one side of a lesion to the other, passing through the center, which occurs after RF. A clear relationship with tumor recurrence has not been demonstrated and it is probably ascribable to non-tumor vessels. A careful observation of these patients is suggested [56].

The capability of CEUS to explore a mass in three dimensions has stimulated radiologists to explore its utility in guiding transarterial chemoembolization (TACE), a procedure usually 


\section{Liver

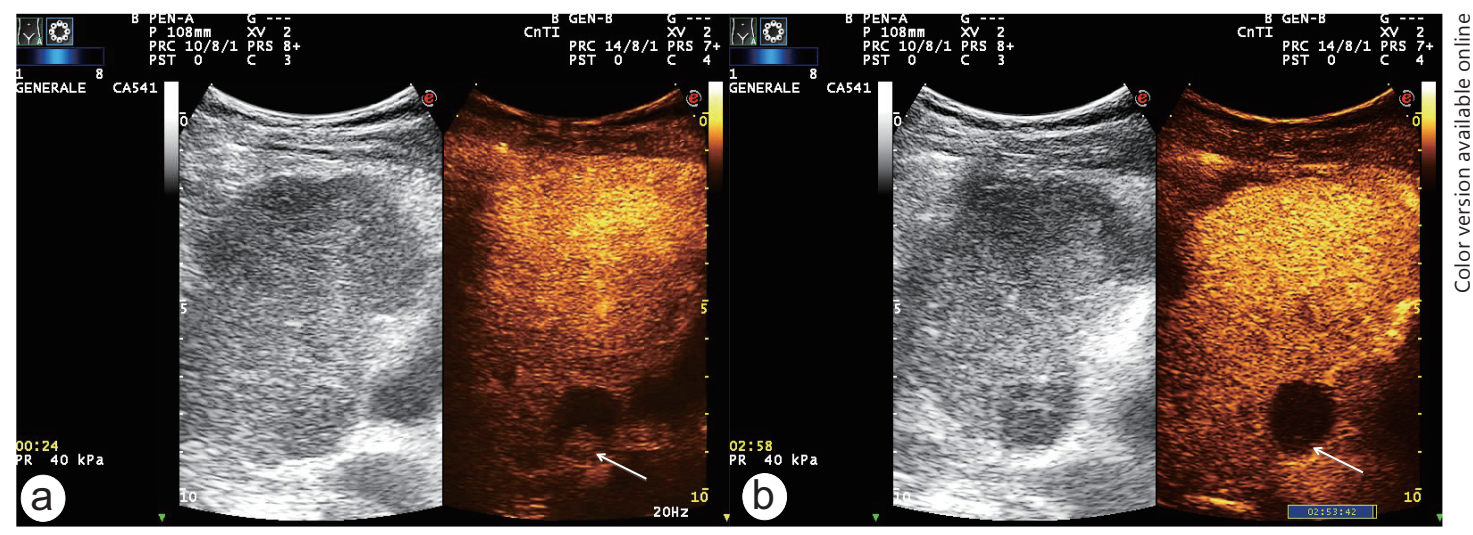

Fig. 2. CEUS examination one month after TACE for HCC. In (a), intratumoral localized enhancement in the arterial phase is evident in the lower part of the nodule followed by wash-out (b) in the late phase, a pattern indicative of limited residual tumor viability.

directed by angiography. An incomplete embolization may occur in cases of extrahepatic feeding arteries, or when additional hepatic feeders distal to the super-selective catheter position are present. Indeed, when nonenhancing areas are located anteriorly or posteriorly, they are not appreciable with two-dimensional angiography. Burgmans et al. [57] proposed injecting microbubbles under US guidance directly through the intraarterial catheter in order to have a multiplanar visualization before TACE. A modification in the treatment approach occurred in $44.4 \%$ of cases due to the additional information provided by this technique, which was consistent with catheter-directed CT arteriography, used as a reference for intrahepatic findings.

A possible role in the evaluation of TACE results has also been tested (fig. 2) [58]. Although CT can evaluate more lesions at the same time, being a panoramic technique, it suffers from the beam-hardening artifacts of Lipiodol ${ }^{\circledR}$ in the instance of conventional TACE. Such artifacts negatively affect the sensitivity in detecting residual tumor viability. Conversely, CEUS is not affected by Lipiodol ${ }^{\circledR}$ artifacts, but it allows the evaluation of only one or two lesions for each contrast injection, and visualization of deeply located lesions may be unfeasible. Multiplicity, hypoenhancement on pretreatment CEUS and infiltrative diffuse growth pattern are tumor conditions that affect CEUS efficacy. Indeed, CEUS was reported to provide the accurate assessment of treatment response to locoregional therapies using one contrast injection in $85 \%$ of solitary tumors, $58 \%$ in the case of two treated tumors and in $67 \%$ in the case of three treated tumors [59], respectively. In case of the absence of the aforementioned limitations, concordance with CT/MRI is instead extremely high. Cases of residual activity detected only at CEUS and overlooked at CT or MRI have been reported [56]. A high specificity in detecting residual activity at 24 hours has been demonstrated with fusion imaging with CT [60]. Taken together, these results indicate that CEUS cannot replace CT or MRI in the assessment of response to TACE, but that it can be valuably integrated with other imaging techniques in difficult cases.

Lastly, the increasing supply of antiangiogenic treatments that are under investigations and that probably will be available in the near future underlines the importance of an early assessment of tumor response to drugs. These molecules can be effective even in absence of tumor shrinkage, making purely dimensional criteria unsuitable to define the response to treatment. A cystic transformation often combined with tumor enlargement may occur in cases of responder tumors [61]. However, these finding are not accurate, making mandatory the use of contrast to appreciate active areas. An eventual decrease in tumor perfusion can be assessed visually or through imaging techniques able to quantify the enhancement. In par- 


\section{Liver Cancer}

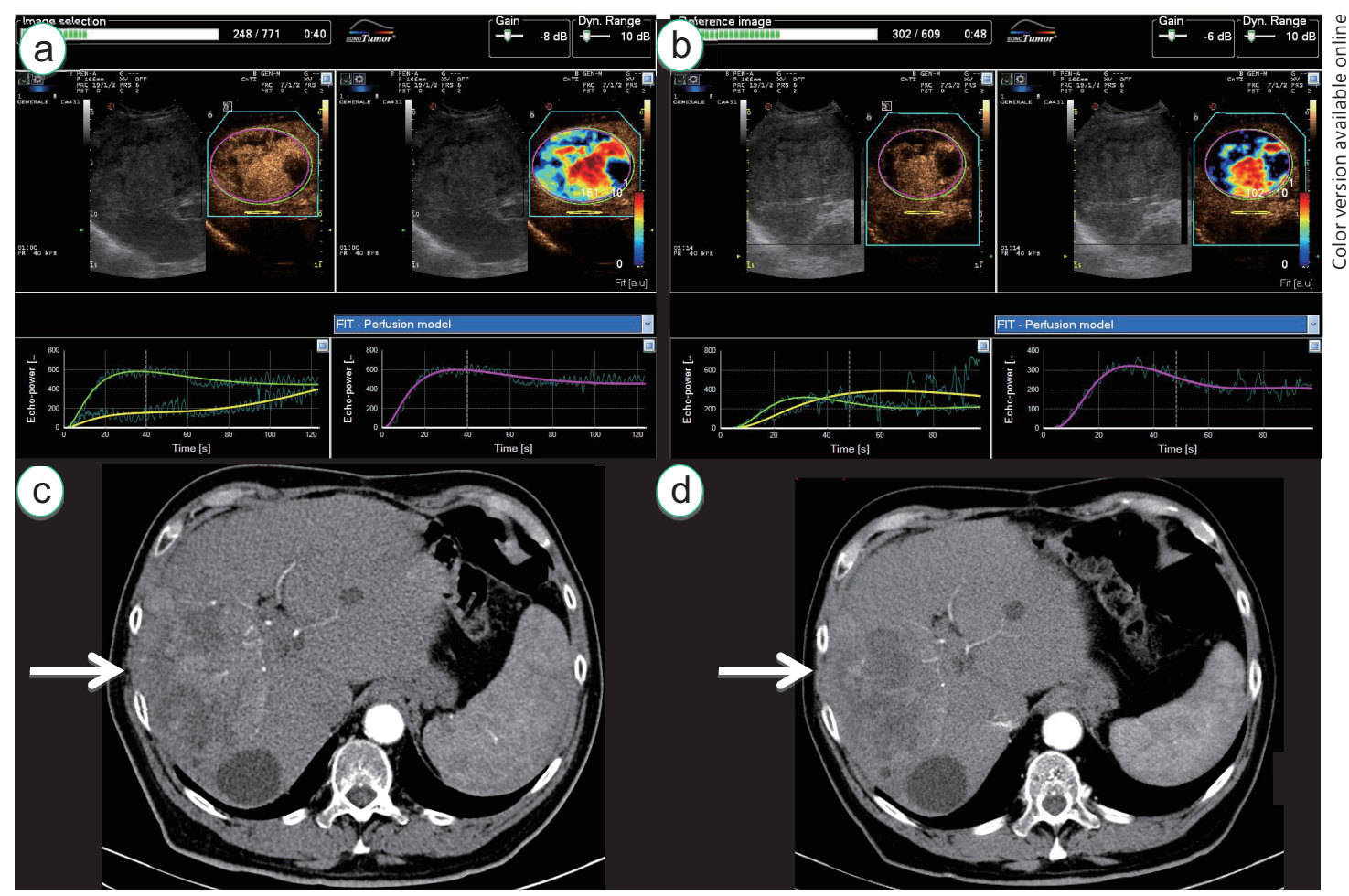

Fig. 3. Quantification of enhancement under antiangiogenenic treatment. US contrast enhancement quantification, using a dedicated software before starting sorafenib treatment (a) and at two weeks posttreatment (b) indicates a reduction in the arterial enhancement and tumor perfusion (see curves under the ultrasound images). CT examination performed at baseline (c; the arrow indicates the active HCC and is the target of systemic treatment) and after two months of treatment (d) demonstrates stable disease according to dimensional criteria, with some increase of the nonperfused hypodense areas.

ticular, general imaging software or dedicated packages can be used which are specifically developed to this aim and able to create time intensity curves of enhancement in a selected region (fig. 3). Furthermore, with the use of such software, raw data (uncompressed data before video-visualization) can be analyzed, which is essential for the most accurate analysis due to their linear behavior [62].

The EFSUMB has recently published advice about dynamic CEUS contrast quantification, showing great appeal of this technique [63]. A milestone is represented by the study conducted by Lassau et al. [64] that included 539 patients with different tumors including HCCs in 19 French centers. It demonstrated that the area under the curve (AUC) of tumor enhancement after a single injection of SonoVue ${ }^{\circledR}$ at day 30 with respect to the AUC at day 0 could be useful to predict the progression and survival from antiangiogenic treatment. Interestingly, only the ratio emerged as significant and not the absolute value. The percentage changes of the AUC both at day 15 and at day 30 with respect to baseline have been confirmed as predictive of response in another series of 28 HCC patients [65]. Other parameters including peak intensity, time to peak and slope coefficient of wash-in significantly differed between responders and nonresponders on the basis of m-RECIST criteria by CT performed at two months. On the other hand, a third study [66] emphasized time to peak at one month as the best discriminator for responders and nonresponders, a parameter that continued to increase in the responder group and that indicates the impact of antiangiogenetic treatments on dynamic flow criteria. Moreover, time to peak in their series suggested a longer median survival of 273 days in the responder group versus 214 days in the nonre- 
sponder group. However, it seems that the presence of portal vein thrombosis influences hemodynamic parameters, making it mandatory to identify it during the quantification process. Indeed, in cases of portal vein thrombosis, faster rise time and time to peak and higher maximum intensity have been reported [67]. Using CEUS in a preclinical animal model, we recently demonstrated that the response to sorafenib can be restored after transient interruption of treatment, whereas definitive treatment withdrawal leads to a rebound increase in vascular endothelial growth factor receptor-2 [68].

The future will probably and hopefully include targeted CEUS, giving the ability to the physician to go beyond the simple perfusion appearance and to explore FLLs on a molecular basis. Indeed, potentially non-immunogenic targeted microbubbless have been developed (BR55 ${ }^{\circledR}$; Bracco Imaging, Switzerland). So far, the only human applications include an experimental phase 0 study on prostate cancer, but preclinical results are very promising [69], especially in the field of response to antiangiogenic treatment. However, once approved for clinical use, molecular CEUS may improve HCC diagnosis or residual activity detection after locoregional treatments, widening the horizons of CEUS.

In conclusion, the extreme manageability of CEUS allows its use in several fields of HCC diagnosis and treatment, including planning and evaluation of the response. However, a knowledge of its limitations is important in order to exploit its advantages without affecting patient management.

\section{Disclosure Statement}

F.B. declares the following conflicts of interest: Bayer, speaker and advisory board fees, Bracco, speaker fee, Esaote, research contract. The other authors have nothing to declare.

\section{References}

1 Albrecht T, Blomley M, Bolondi L, Claudon M, Correas JM, Cosgrove D, Greiner L, Jäger K, Jong ND, Leen E, Lencioni R, Lindsell D, Martegani A, Solbiati L, Thorelius L, Tranquart F, Weskott HP, Whittingham T, EFSUMB Study Group: Guidelines for the use of contrast agents in ultrasound. January 2004. Ultraschall Med 2004;25:249-256.

2 Salvatore V, Borghi A, Piscaglia F: Contrast-enhanced ultrasound for liver imaging: recent advances. Curr Pharm Des 2012;18:2236-2252.

3 Gaiani S, Celli N, Piscaglia F, Cecilioni L, Losinno F, Giangregorio F, Mancini M, Pini P, Fornari F, Bolondi L: Usefulness of contrast-enhanced perfusional sonography in the assessment of hepatocellular carcinoma hypervascular at spiral computed tomography. J Hepatol 2004;41:421-426.

4 Bruix J, Sherman M, Practice Guidelines Committee, American Association for the Study of Liver Diseases: Management of hepatocellular carcinoma. Hepatology 2005;42:1208-1236.

5 Ueda K, Terada T, Nakanuma Y, Matsui O: Vascular supply in adenomatous hyperplasia of the liver and hepatocellular carcinoma: a morphometric study. Hum Pathol 1992;23:619-626.

6 Hayashi M, Matsui O, Ueda K, Kawamori Y, Kadoya M, Yoshikawa J, Gabata T, Takashima T, Nonomura A, Nakanuma Y: Correlation between the blood supply and grade of malignancy of hepatocellular nodules associated with liver cirrhosis: evaluation by CT during intraarterial injection of contrast medium. AJR Am J Roentgenol 1999;172:969-976.

7 Matsui 0: Imaging of multistep human hepatocarcinogenesis by CT during intra-arterial contrast injection Intervirology 2004;47:271-276.

8 Kudo M: Early hepatocellular carcinoma: definition and diagnosis. Liver Cancer 2013;2:69-72.

9 Kudo M: Malignant transformation of hepatocellular adenoma: how frequently does it happen? Liver Cancer 2015;4:1-5

10 Bruix J, Sherman M, Llovet JM, Beaugrand M, Lencioni R, Burroughs AK, Christensen E, Pagliaro L, Colombo M, Rodés J, EASL Panel of Experts on HCC European Association for the Study of the Liver: Clinical management of hepatocellular carcinoma. Conclusions of the Barcelona-2000 EASL conference. J Hepatol 2001;35:421-430. 
11 Claudon M, Cosgrove D, Albrecht T, Bolondi L, Bosio M, Calliada F, Correas JM, Darge K, Dietrich C, D’Onofrio M, Evans DH, Filice C, Greiner L, Jäger K, Jong N, Leen E, Lencioni R, Lindsell D, Martegani A, Meairs S, Nolsøe C, Piscaglia F, Ricci P, Seidel G, Skjoldbye B, Solbiati L, Thorelius L, Tranquart F, Weskott HP, Whittingham T: Guidelines and good clinical practice recommendations for contrast enhanced ultrasound (CEUS) - update 2008. Ultraschall Med 2008;29:28-44.

12 Forner A, Vilana R, Ayuso C, Bianchi L, Solé M, Ayuso JR, Boix L, Sala M, Varela M, Llovet JM, Brú C, Bruix J: Diagnosis of hepatic nodules $20 \mathrm{~mm}$ or smaller in cirrhosis: Prospective validation of the noninvasive diagnostic criteria for hepatocellular carcinoma. Hepatology 2008;47:97-104.

13 Sangiovanni A, Manini MA, Iavarone M, Romeo R, Forzenigo LV, Fraquelli M, Massironi S, Della Corte C, Ronchi G, Rumi MG, Biondetti P, Colombo M: The diagnostic and economic impact of contrast imaging techniques in the diagnosis of small hepatocellular carcinoma in cirrhosis. Gut 2010;59:638-644.

14 Makuuchi M, Kokudo N, Arii S, Futagawa S, Kaneko S, Kawasaki S, Matsuyama Y, Okazaki M, Okita K, Omata M, Saida Y, Takayama T, Yamaoka Y: Development of evidence-based clinical guidelines for the diagnosis and treatment of hepatocellular carcinoma in Japan. Hepatol Res 2008;38:37-51.

15 Omata M, Lesmana LA, Tateishi R, Chen PJ, Lin SM, Yoshida H, Kudo M, Lee JM, Choi BI, Poon RT, Shiina S, Cheng AL, Jia JD, Obi S, Han KH, Jafri W, Chow P, Lim SG, Chawla YK, Budihusodo U, Gani RA, Lesmana CR, Putranto TA, Liaw YF, Sarin SK: Asian Pacific Association for the Study of the Liver consensus recommendations on hepatocellular carcinoma. Hepatol Int 2010;4:439-474.

16 Bruix J, Sherman M American Association for the Study of Liver Diseases: Management of hepatocellular carcinoma: an update. Hepatology 2011;53:1020-1022.

17 Bolondi L, Gaiani S, Celli N, Golfieri R, Grigioni WF, Leoni S, Venturi AM, Piscaglia F: Characterization of small nodules in cirrhosis by assessment of vascularity: the problem of hypovascular hepatocellular carcinoma. Hepatology 2005;42:27-34.

18 Nicolau C, Catalá V, Vilana R, Gilabert R, Bianchi L, Solé M, Pagés M, Brú C: Evaluation of hepatocellular carcinoma using SonoVue, a second generation ultrasound contrast agent: correlation with cellular differentiation. Eur Radiol 2004;14:1092-1099.

19 Fan ZH, Chen MH, Dai Y, Wang YB, Yan K, Wu W, Yang W, Yin SS: Evaluation of primary malignancies of the liver using contrast-enhanced sonography: correlation with pathology. AJR Am J Roentgenol 2006;186:1512-1519.

20 Jang HJ, Kim TK, Burns PN, Wilson SR: Enhancement patterns of hepatocellular carcinoma at contrastenhanced US: comparison with histologic differentiation. Radiology 2007;244:898-906.

21 Piscaglia F, Leoni S, Cabibbo G, Borghi A, Imbriaco G, Golfieri R, Bolondi L: Cost analysis of recall strategies for non-invasive diagnosis of small hepatocellular carcinoma. Dig Liver Dis 2010;42:729-734.

22 Ryu SW, Bok GH, Jang JY, Jeong SW, Ham NS, Kim JH, Park EJ, Kim JN, Lee WC, Shim KY, Lee SH, Kim SG, Cha SW, Kim YS, Cho YD, Kim HS, Kim BS: Clinically useful diagnostic tool of contrast enhanced ultrasonography for focal liver masses: comparison to computed tomography and magnetic resonance imaging. Gut Liver 2014;8:292-297.

23 Strobel D, Seitz K, Blank W, Schuler A, Dietrich CF, von Herbay A, Friedrich-Rust M, Bernatik T: Tumorspecific vascularization pattern of liver metastasis, hepatocellular carcinoma, hemangioma and focal nodular hyperplasia in the differential diagnosis of 1,349 liver lesions in contrast-enhanced ultrasound (CEUS). Ultraschall Med 2009;30:376-382.

24 Friedrich-Rust M, Klopffleisch T, Nierhoff J, Herrmann E, Vermehren J, Schneider MD, Zeuzem S, Bojunga J: Contrast-Enhanced Ultrasound for the differentiation of benign and malignant focal liver lesions: a metaanalysis. Liver Int. 2013 33:739-55.

25 Yang H, Liu GJ, Lu MD, Xu HX, Xie XY: Evaluation of the vascular architecture of focal liver lesions using micro flow imaging. J Ultrasound Med 2013;32:1157-1171.

26 Xu HX, Lu MD, Liu LN, Zhang YF, Guo LH, Xu JM, Liu C: Discrimination between neoplastic and non-neoplastic lesions in cirrhotic liver using contrast-enhanced ultrasound. Br J Radiol 2012;85:1376-1384.

27 Ikeda K, Saitoh S, Koida I, Tsubota A, Arase Y, Chayama K, Kumada H: Diagnosis and follow-up of small hepatocellular carcinoma with selective intraarterial digital subtraction angiography. Hepatology 1993;17:1003-1007.

28 Giorgio A, Ferraioli G, Tarantino L, de Stefano G, Scala V, Scarano F, Coppola C, Del Viscovo L: Contrastenhanced sonographic appearance of hepatocellular carcinoma in patients with cirrhosis: comparison with contrast-enhanced helical CT appearance. AJR Am J Roentgenol 2004;183:1319-1326.

29 Quaia E, De Paoli L, Angileri R, Pizzolato R, Cabibbo B, Cova MA: Evidence of diagnostic enhancement pattern in hepatocellular carcinoma nodules $\leq 2 \mathrm{~cm}$ according to the AASLD/EASL revised criteria. Abdom Imaging 2013;38:1245-1253.

30 Forner A, Vilana R, Bianchi L, Rodríguez-Lope C, Reig M, García-Criado MA, Rimola J, Solé M, Ayuso C, Bru C, Bruix J: Lack of arterial hypervascularity at contrast-enhanced ultrasound should not define the priority for diagnostic work-up of nodules $<2 \mathrm{~cm}$. J Hepatol 2015;62:150-155.

31 Iavarone M, Sangiovanni A, Forzenigo LV, Massironi S, Fraquelli M, Aghemo A, Ronchi G, Biondetti P, Roncalli M, Colombo M: Diagnosis of hepatocellular carcinoma in cirrhosis by dynamic contrast imaging: the importance of tumor cell differentiation. Hepatology 2010;52:1723-1730.

32 Boozari B, Soudah B, Rifai K, Schneidewind S, Vogel A, Hecker H, Hahn A, Schlue J, Dietrich CF, Bahr MJ, Kubicka S, Manns MP, Gebel M: Grading of hypervascular hepatocellular carcinoma using late phase of contrast enhanced sonography - a prospective study. Dig Liver Dis 2011;43:484-490. 
33 Liu GJ, Xu HX, Lu MD, Xie XY, Xu ZF, Zheng YL, Liang JY: Correlation between enhancement pattern of hepatocellular carcinoma on real-time contrast-enhanced ultrasound and tumour cellular differentiation on histopathology. Br J Radiol 2007;80:321-330.

34 Ariizumi S, Takasaki K, Yamamoto M, Ohtsubo T, Katsuragawa H, Katagiri S: Histopathologic differentiation of the main nodule determines outcome after hepatic resection for synchronous multicentric hepatocellular carcinomas. Hepatogastroenterology 2004;51:500-504.

35 Vilana R, Forner A, Bianchi L, García-Criado A, Rimola J, de Lope CR, Reig M, Ayuso C, Brú C, Bruix J: Intrahepatic peripheral cholangiocarcinoma in cirrhosis patients may display a vascular pattern similar to hepatocellular carcinoma on contrast-enhanced ultrasound. Hepatology 2010;51:2020-2029.

36 Salvatore V, Bolondi L: Clinical impact of ultrasound-related techniques on the diagnosis of focal liver lesions. Liver Cancer 2012;1:238-246.

37 Lu Q, Xue LY, Wang WP, Huang BJ, Li CX: Dynamic enhancement pattern of intrahepatic cholangiocarcinoma on contrast-enhanced ultrasound: the correlation with cirrhosis and tumor size. Abdom Imaging 2015; (in press).

38 Yuan MX, Li R, Zhang XH, Tang CL, Guo YL, Guo DY, Luo MK: Factors affecting the enhancement patterns of intrahepatic cholangiocarcinoma (ICC) on contrast-enhanced ultrasound (CEUS) and their pathological correlations in patients with single lesion. Ultraschall Med 2015 Apr 28. [Epub ahead of print] doi:10.1055/s-0034-1399485

39 Li R, Yuan MX, Ma KS, Li XW, Tang CL, Zhang XH, Guo DY, Yan XC: Detailed analysis of temporal features on contrast enhanced ultrasound may help differentiate intrahepatic cholangiocarcinoma from hepatocellular carcinoma in cirrhosis. PLoS ONE 2014;9:e98612.

40 Galassi M, Iavarone M, Rossi S, Bota S, Vavassori S, Rosa L, Leoni S, Venerandi L, Marinelli S, Sangiovanni A, Veronese L, Fraquelli M, Granito A, Golfieri R, Colombo M, Bolondi L, Piscaglia F: Patterns of appearance and risk of misdiagnosis of intrahepatic cholangiocarcinoma in cirrhosis at contrast enhanced ultrasound. Liver Int 2013;33:771-779.

41 European Association For The Study Of The Liver European Organisation For Research And Treatment Of Cancer: EASL-EORTC clinical practice guidelines: management of hepatocellular carcinoma. J Hepatol 2012;56:908-943.

42 Manini MA, Sangiovanni A, Fornari F, Piscaglia F, Biolato M, Fanigliulo L, Ravaldi E, Grieco A, Colombo M, Study Participants: Clinical and economical impact of 2010 AASLD guidelines for the diagnosis of hepatocellular carcinoma. J Hepatol 2014;60:995-1001.

43 SonoVue (sulphur hexafluoride microbubbles) - contrast agent for contrast enhanced ultrasound imaging of the liver. 2012; http://publications.nice.org.uk/dg5.

44 Bolondi L, Cillo U, Colombo M, Craxì A, Farinati F, Giannini EG, Golfieri R, Levrero M, Pinna AD, Piscaglia F, Raimondo G, Trevisani F, Bruno R, Caraceni P, Ciancio A, Coco B, Fraquelli M, Rendina M, Squadrito G, Toniutto P, Italian Association for the Study of the Liver (AISF) AISF Expert Panel AISF Coordinating Committee: Position paper of the Italian Association for the Study of the Liver (AISF): the multidisciplinary clinical approach to hepatocellular carcinoma. Dig Liver Dis 2013;45:712-723.

45 Lee MW, Lim HK, Kim YJ, Choi D, Kim YS, Lee WJ, Cha DI, Park MJ, Rhim H: Percutaneous sonographically guided radio frequency ablation of hepatocellular carcinoma: causes of mistargeting and factors affecting the feasibility of a second ablation session. J Ultrasound Med 2011;30:607-615.

46 Kim PN, Choi D, Rhim H, Rha SE, Hong HP, Lee J, Choi JI, Kim JW, Seo JW, Lee EJ, Lim HK: Planning ultrasound for percutaneous radiofrequency ablation to treat small $(\leq 3 \mathrm{~cm})$ hepatocellular carcinomas detected on computed tomography or magnetic resonance imaging: a multicenter prospective study to assess factors affecting ultrasound visibility. J Vasc Interv Radiol 2012;23:627-634.

47 Liu F, Yu X, Liang P, Cheng Z, Han Z, Dong B: Contrast-enhanced ultrasound-guided microwave ablation for hepatocellular carcinoma inconspicuous on conventional ultrasound. Int J Hyperthermia 2011;27:555562.

48 Kim AY, Lee MW, Rhim H, Cha DI, Choi D, Kim YS, Lim HK, Cho SW: Pretreatment evaluation with contrastenhanced ultrasonography for percutaneous radiofrequency ablation of hepatocellular carcinomas with poor conspicuity on conventional ultrasonography. Korean J Radiol 2013;14:754-763.

49 Rajesh S, Mukund A, Arora A, Jain D, Sarin SK: Contrast-enhanced US-guided radiofrequency ablation of hepatocellular carcinoma. J Vasc Interv Radiol 2013;24:1235-1240.

50 Sala M, Llovet JM, Vilana R, Bianchi L, Solé M, Ayuso C, Brú C, Bruix J, Barcelona Clínic Liver Cancer Group: Initial response to percutaneous ablation predicts survival in patients with hepatocellular carcinoma. Hepatology 2004;40:1352-1360.

51 Sparchez Z, Radu P, Anton O, Socaciu M, Badea R: Contrast enhanced ultrasound in assessing therapeutic response in ablative treatments of hepatocellular carcinoma. J Gastrointestin Liver Dis 2009;18:243-248.

52 Miyamoto N, Hiramatsu K, Tsuchiya K, Sato Y: Contrast-enhanced sonography-guided radiofrequency ablation for the local recurrence of previously treated hepatocellular carcinoma undetected by B-mode sonography. J Clin Ultrasound 2010;38:339-345.

53 Salvaggio G, Campisi A, Lo Greco V, Cannella I, Meloni MF, Caruso G: Evaluation of posttreatment response of hepatocellular carcinoma: comparison of ultrasonography with second-generation ultrasound contrast agent and multidetector CT. Abdom Imaging 2010;35:447-453.

54 Kisaka Y, Hirooka M, Kumagi T, Uehara T, Hiasa Y, Kumano S, Tanaka H, Michitaka K, Horiike N, Mochizuki $\mathrm{T}$, Onji M: Usefulness of contrast-enhanced ultrasonography with abdominal virtual ultrasonography in assessing therapeutic response in hepatocellular carcinoma treated with radiofrequency ablation. Liver Int 2006;26:1241-1247. 
55 Shimizu M, Iijima H, Horibe T, Yamada M, Suzuki S, Yanagisawa K, Seki T, Moriyasu F: Usefulness of contrast-enhanced ultrasonography with a new contrast mode, Agent Detection Imaging, in evaluating therapeutic response in hepatocellular carcinoma treated with radio-frequency ablation therapy. Hepatol Res 2004;29:235-242.

56 Takahashi M, Maruyama H, Shimada T, Kamezaki H, Okabe S, Kanai F, Yoshikawa M, Yokosuka O: Linear enhancement after radio-frequency ablation for hepatocellular carcinoma: is it a sign of recurrence? Ultrasound Med Biol 2012;38:1902-1910.

57 Burgmans MC, van Erkel AR, Too CW, Coenraad M, Lo RH, Tan BS: Pilot study evaluating catheter-directed contrast-enhanced ultrasound compared to catheter-directed computed tomography arteriography as adjuncts to digital subtraction angiography to guide transarterial chemoembolization. Clin Radiol 2014;69:1056-1061.

58 Kaufmann S, Schulze M, Spira D, Horger M: Comparison of volume perfusion computed tomography and contrast-enhanced ultrasound for assessment of therapeutic effect of transarterial chemoembolization in patients with hepatocellular carcinoma: a preliminary report. Acta Radiol 2015; (in press).

59 Moschouris H, Malagari K, Papadaki MG, Kornezos I, Gkoutzios P, Tepelenis N, Matsaidonis D: Short-term evaluation of liver tumors after transarterial chemoembolization: limitations and feasibility of contrastenhanced ultrasonography. Abdom Imaging 2011;36:718-728.

60 Wobser H, Wiest R, Salzberger B, Wohlgemuth WA, Stroszczynski C, Jung EM: Evaluation of treatment response after chemoembolisation (TACE) in hepatocellular carcinoma using real time image fusion of contrast-enhanced ultrasound (CEUS) and computed tomography (CT)-preliminary results. Clin Hemorheol Microcirc 2014;57:191-201.

61 Moschouris H, Malagari K, Gkoutzios P, Kalokairinou M, Stamatiou K, Chatzimichail K, Kornezos I, Karagiannis E, Kiltenis M, Papadaki MG: Intermediate and advanced hepatocellular carcinoma treated with the antiangiogenic agent sorafenib. Evaluation with unenhanced and contrast-enhanced ultrasonography. Med Ultrasound 2012;14:87-94.

62 Gauthier M, Leguerney I, Thalmensi J, Chebil M, Parisot S, Peronneau P, Roche A, Lassau N: Estimation of intra-operator variability in perfusion parameter measurements using DCE-US. World J Radiol 2011;3:70-81.

63 Dietrich CF, Averkiou MA, Correas JM, Lassau N, Leen E, Piscaglia F: An EFSUMB introduction into Dynamic Contrast-Enhanced Ultrasound (DCE-US) for quantification of tumour perfusion. Ultraschall Med 2012;33:344-351.

64 Lassau N, Bonastre J, Kind M, Vilgrain V, Lacroix J, Cuinet M, Taieb S, Aziza R, Sarran A, Labbe-Devilliers C, Gallix B, Lucidarme O, Ptak Y, Rocher L, Caquot LM, Chagnon S, Marion D, Luciani A, Feutray S, Uzan-Augui J, Coiffier B, Benastou B, Koscielny S: Validation of dynamic contrast-enhanced ultrasound in predicting outcomes of antiangiogenic therapy for solid tumors: the French multicenter support for innovative and expensive techniques study. Invest Radiol 2014;49:794-800.

65 Zocco MA, Garcovich M, Lupascu A, Di Stasio E, Roccarina D, Annicchiarico BE, Riccardi L, Ainora ME, Ponziani F, Caracciolo G, Rapaccini GL, Landolfi R, Siciliano M, Pompili M, Gasbarrini A: Early prediction of response to sorafenib in patients with advanced hepatocellular carcinoma: the role of dynamic contrast enhanced ultrasound. J Hepatol 2013;59:1014-1021.

66 Knieling F, Waldner MJ, Goertz RS, Zopf S, Wildner D, Neurath MF, Bernatik T, Strobel D: Early response to anti-tumoral treatment in hepatocellular carcinoma-can quantitative contrast-enhanced ultrasound predict outcome? Ultraschall Med 2013;34:38-46.

67 Wang Z, Liu G, Lu MD, Xie X, Kuang M, Wang W, Xu Z, Lin M, Chen L: Role of portal vein tumor thrombosis in quantitative perfusion analysis of contrast-enhanced ultrasound of hepatocellular carcinoma. Ultrasound Med Biol 2015;41:1277-1286.

68 Marinelli S, Salvatore V, Baron Toaldo M, Milazzo M, Croci L, Venerandi L, Pecorelli A, Palamà C, Diana A, Bolondi L, Piscaglia F: Evaluation of the impact of transient interruption of antiangiogenic treatment using ultrasound-based techniques in a murine model of hepatocellular carcinoma. BMC Cancer 2014;14:403-411.

69 Baron Toaldo M, Salvatore V, Marinelli S, Palamà C, Milazzo M, Croci L, Venerandi L, Cipone M, Bolondi L, Piscaglia F: Use of VEGFR-2 targeted ultrasound contrast agent for the early evaluation of response to sorafenib in a mouse model of hepatocellular carcinoma. Mol Imaging Biol 2015;17:29-37. 